\section{Canine aural hematoma: some thoughts leading to a new treatment - internal drainage}

\section{Henning Horstmann}

Dalum Dyreklinik, Odense, Denmark

For many years canine aural hematomas have been treated using a combination of systemic and local corticosteroids. This treatment has proved to be quite efficient. However the mechanism of the action of the steroids has not been fully understood. This of course is related to the fact, that the etiology of aural hematomas is still uncertain. The theory of trauma as the main reason has for a long time been predominant. This theory however does not really explain any of the main findings in the typical patient.

These symptoms on the other hand could be explained by a theory of a hemodynamic disorder. White blood cells need to adapt their shape in order to pass the capillaries. A decrease in this ability in combination with instability of capillaries leads to leaking of plasma in an amount exceeding the capacity of reabsorption.

A coincidental finding of a dog with an atypical swelling of an earlobe led to the conclusion, that when we are dealing with canine aural hematomas, we are actually facing two problems. First, what is causing the hematoma, and it was concluded, that the cartilage plays a major role for the hematoma to occur. The second problem is how to get rid of the extra vascular fluid, and it was concluded, that if the fluid could gain access to the venous drainage on the outside of the earlobe, then reabsorption could be improved.

The next dog with aural hematoma was treated with steroids for a few days and then anesthesised. Via a small skin incision a hole was made in the cartilage using a biopsy punch. The fluid was evacuated and the skin closed with 2 sutures.

This treatment is easy to perform. It seems quite efficient and has 2 more benefits. It reduces the risk of recurrence. If recurrence appears it can probably be treated with systemic steroids. The second benefit is that it seems to reduce the risk of deformation of the earlobe.

\section{The efficacy of sarolaner} (Simparica'⿳一 ${ }^{\mathrm{TM}}$ chewable tablets) for the treatment of induced flea infestations compared to afoxolaner (NexGard ${ }^{\circledR}$ chewable tablets), spinosad + millbemycine (Trifexis ${ }^{\circledR}$ chewable tablets) and fipronil + methoprene (Frontline Plus ${ }^{\circledR}$ spot-on) in dogs

\section{Csilla Becskei', Melanie Myers², Sara Chapin ${ }^{2}$, Nicole Honsberger ${ }^{2}$, Sean P. Mahabir ${ }^{2}$, Robert H. Six ${ }^{2}$ \\ 1 Zoetis, Zaventem, Belgium \\ 2 Zoetis, Kalamazoo, MI, USA}

The speed of kill of sarolaner (Simparica ${ }^{\mathrm{TM}}$ ) against fleas was compared with afoxolaner (NexGard $\left.{ }^{\circledR}\right)$, spinosad + milbemycin $\left(\right.$ Trifexis $^{\circledR}$ ) and fipronil + methoprene (Frontline Plus ${ }^{\circledR}$ Spot-On) in three separate, randomised, placebo-controlled, double-blinded studies in dogs.

In each study, three groups of dogs received placebo or sarolaner orally or the comparator product administered according to their respective label instructions once on Day 0 ( $n=8 /$ group). Dogs were infested with 100 fleas (Ctenocephalides felis) on days $-1,7,14,21,28$, and 35 . Percent reduction in mean live flea counts (efficacy) was determined for separate groups at 8, 12 and 24 hours after treatment and after each post-treatment infestation versus placebo. A mixed linear model for repeated measures was used to compare flea counts between treatment groups.

Against existing flea infestations, sarolaner produced $100 \%$ efficacy within 8 hours in two studies and $>99 \%$ efficacy in the third. Sarolaner provided $>99$ to $100 \%$ efficacy within 12 hours for at least 4 weeks and all sarolaner treated dogs were flea-free in all three studies within 24 hours for 35 days after treatment, except in one study where one single flea was found on one dog on Day 8. The oral products killed almost all fleas within 8 hours after treatment, while fipronil + methoprene spot-on only had an effect 12 hours after treatment. Sarolaner provided better speed of kill within 8 hours during the entire treatment interval than oral afoxolaner and from three to five weeks after treatment compared to spinosad+milbemycine.

Sarolaner killed significantly more fleas at all 8 hour time points than afoxolaner and at the 12 hour time points on days 28 and 35 . Sarolaner reduced flea counts significantly more than spinosad + milbemycine 8 hours after re-infestations on days 21 and 28 and at all time points on Day 35. Sarolaner provided significantly better efficacy than fipronil+methoprene 8 and 12 hours after treatment. Fipronil+methoprene killed significantly more fleas on a single day, Day 7, at the 8 hour time point. 